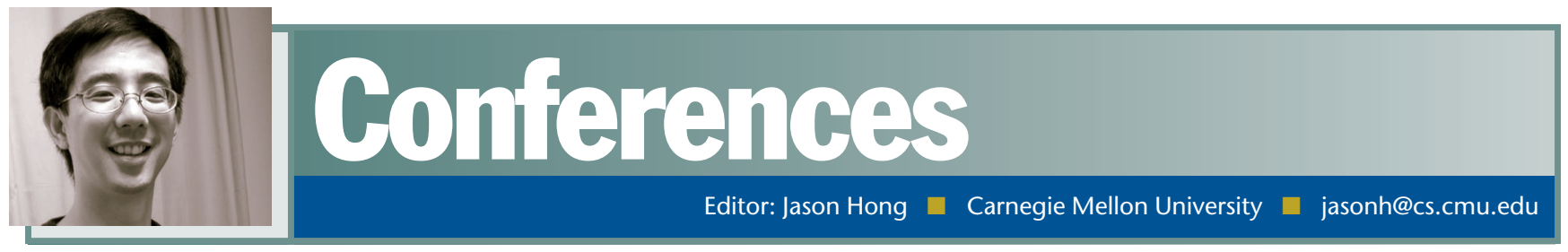

\title{
HotMobile 2008: Postconference Report
}

\author{
Janne Lindqvist, Pravin Pawar, and Erich Stuntebeck
}

T he 9th Workshop on Mobile Computing Systems and Applications (HotMobile 08) took place in February in the heart of Napa Valley, California's wine country. Sponsored by ACM Sigmobile for the first time, the workshop attracted approximately 75 mobile-computing researchers. As with other HotTopic workshops, the organizers intend the workshop to evolve as a testbed for ideas prior to submission to the annual International Conference on Mobile Systems, Applications, and Services (MobiSys), held in June 2008. In fact, many members of the MobiSys program committee attended the workshop because their meeting took place after the workshop.

This year, the HotMobile committee accepted 18 of the 79 submitted papers (a 23 percent acceptance rate). Along with the paper presentations and stimulating, intense discussions, the HotMobile 2008 workshop also featured a keynote address, posters and demo sessions, and a doctoral consortium.

\section{THE MOBILE MIRACLE}

Mark Corner (University of Massachusetts, Amherst) opened the workshop and introduced the keynote speaker, B.J. Fogg, founder of the Stanford Persuasive Technology Lab.

Fogg began his talk "The Mobile Miracle" with the claim that the mobile device is the most amazing invention in history and that it has had the largest impact on humankind. Because mobile phones combine mobile devices with ongoing developments in software and communication technologies, they have the potential to change the way people think and act in the following ways:

\section{We might use mobile devices to persuade people to enhance the quality of life and eventually even to further world peace.}

- Concierge. Mobile devices can respond with help when needed-for example, in hotel searching.

- Coach. Mobile phones can track our goals and context. When the time is right, the mobile coach prompts us to take an action-for example, reminding us about tasks.

- Court jester. Mobile phones can amuse us with games, fun, information, and flirty social interactions.

Fogg then argued that although today's social software is designed for desktop experience, the real show will begin when mobile phones become a viable platform for Web 2.0 technolo- gies. As an analogy, YouTube has had considerable impact on US elections this year, but mobile devices could perform that role in future elections. Fogg envisions that we might use mobile devices to persuade people to enhance the quality of life for individuals, communities, and society, and eventually even to further world peace.

\section{MAKING SENSE OF SENSORS}

The first paper session focused on interpreting data obtained from sensors. Erich Stuntebeck (Georgia Institute of Technology) presented "HealthSense: Classification of Health-Related Sensor Data through User-Assisted Machine Learning." HealthSense avoids the problem of partial automated detection in some important chronic health conditions, such as pain and depression, by real-time tagging of health-related sensor data using machine-learning techniques. The system, although automated, periodically uses patient input as feedback to improve accuracy and help classify interesting events (for instance, pain or itching).

Deborah Estrin (University of California at Los Angeles) presented her group's research on "Seeing Our Signals: Combining Location Traces and Web-Based Models for Personal Discovery." By combining the power of mobile sensing with Web-based geo- 
spatial data and models, we can begin to see our own signals, the patterns of our daily life as we interact with the world around us. The authors propose a Personal Environmental Impact Report (PEIR) system and demonstrate how we can easily gather location time-series data as an index into geospatial models to infer personal environmental impacts and exposures. Application examples include choice of transportation mode, overall carbon footprint, and opportunities for healthy eating.

Nicholas Lane (Dartmouth College) presented the last paper in the session, "Urban Sensing Systems: Opportunistic or Participatory?" He focused on the precise role of humans in the development of sensing systems for urban deployments. Lane contrasts two endpoints of the spectrum of conscious human involvement-namely, participatory sensing and opportunistic sensing. In contrast to opportunistic systems such as CenceMe, participatory systems such as Twitter assume significant human involvement. Lane presented an evaluation model for these two humaninvolvement approaches and argued that opportunistic sensing more easily supports larger-scale applications and broader diversity within such applications. Several people suggested looking at hybrid systems, combining both participatory and opportunistic approaches, with the presenter agreeing that a bit of input combined with automation could be the way to go.

\section{COMPOSITION AND MODULARITY}

In the second session, Roy Want (Intel Research) presented "Dynamic Composable Computing” (DCC). $\mathrm{He}$ addressed the problem of giving users access to a full personal-computing experience with the mobility that smart phones afford. Want pointed out that the reason he was giving the talk then and not, for example, five years earlier was that the bandwidth of wireless LAN is approaching that of wired networking, and ultra-wideband should have even more. DCC enables impromptu assembly of a logical computer from the best set of wireless component parts available nearby. DCC uses context-aware discovery to find services offered by nearby devices, a composition engine to compose the services, and a user interface. The GUI gives users a representation of the computers discovered and the resources available for composition, along with any existing connections.

Perhaps the most controversial paper in the workshop was "Getting Mod: A Look at Modularity in Mobile System Design." The author, Evan Barba (Georgia Institute of Technology), claims that today's mobile systems have very little modularity. He explained the basics of modularity, presented the benefits of modularity in desktop computers, and then argued why mobile computing isn't modular. One audience member criticized this idea by observing that modularity would increase the cost of already expensive wires and connectors, so economies of scale don't work.

\section{WIRELESS}

The third session looked at topics in wireless technologies. Nathanael Thompson (University of Illinois, UrbanaChampaign) presented " $100 \%$ Certified Organic: Design and Implementation of Self-Sustaining Cellular Networks." Thompson introduced organic networks-cellularlike networks in which end users own and install part of the infrastructure and share it with other customers. The goals are to provide selfmanaged service designs and reduce the cost of customer-installed networks. Thompson described the design and implementation of two basic cellularnetwork services: authentication and location registration. He also highlighted the challenges that researchers must solve to prove that organic networks are a viable alternative to centrally managed cellular infrastructures.

Suman Banerjee (University of Wisconsin, Madison) presented his group's paper "Supporting Continuous Mobility through Multi-rate Wireless
IEEE Computer Society

Publications Office

10662 Los Vaqueros Circle, PO Box 3014 Los Alamitos, CA 90720-1314

STAFF

Lead Editor

Shani Murray

smurray@computer.org

Senior Editorial Services Manager

Crystal R. Shif

Magazine Editorial Manager

Steve Woods

Staff Editors

Brian Brannon, Kathleen Clark-Fisher, Jennifer Gardelle, Jennifer Stout,

Dale Strok, Linda World, and Ed Zintel

Publications Administrator Hilda Carman

pervasive@computer.org

Contributing Editors

Cheryl Baltes, Thomas Centrella,

Glenda McCarthy, and Joan Taylor

Production Editor

Jennie Zhu

Technical Illustrations

Alex Torres

Associate Publisher Dick Price

Membership Manager

Georgann Carter

Senior Business Development Manager Sandra Brown

Senior Advertising Coordinator Marian Anderson
Submissions: Access the IEEE Computer Society's Web-based system, Manuscript Central, at http:// cs-ieee.manuscriptcentral.com/index.html. Be sure to select the right manuscript type when submitting. Articles must be original and should be approximately 5,000 words long, preferably not exceeding 10 references. Visit www.computer.org/ pervasive for editorial guidelines.

Editorial: Unless otherwise stated, bylined articles as well as products and services reflect the author's or firm's opinion; inclusion does not necessarily constitute endorsement by the IEEE Computer Society or the IEEE. 
Packetization." The authors modulated a single packet with multiple rates to gather feedback on the wireless link's quality. This mechanism should result in better adaptation for the quality of the link. A workshop attendee suggested that error-correcting codes are similar, but the differences or benefits aren't clearly understood.

Ranveer Chandra (Microsoft Research) presented "Wi-Fi Neighborcast: Enabling Communication among Nearby Clients." To benefit from the increasing density of open Wi-Fi access points (APs) in urban areas as well as in enterprise and campus networks, Neighborcast enables clients to discover other clients within the APs' range and to communicate with each other even when they're associated with different APs. Neighborcast could ultimately improve AP selection by locating nearby buddies and could perform cooperative problem diagnosis. An attendee raised a concern about multicast application collisions, but Ranveer pointed that few people use multicast.

During his talk on "The Managed Motorway: Real-Time Vehicle Scheduling-A Research Agenda," Vinny Cahill (Trinity College Dublin) described a novel approach to vehicle scheduling based on realtime scheduling, local coordination, and real-time intervehicle communication. In this approach, drivers would reserve variablesized slots on motorway lanes, which would enable enforcement of traveltime guarantees. He then presented a research agenda consisting of real-time communication in dynamic vehicular networks, local real-time coordination, and real-time slot scheduling.

\section{SECURITY}

Kan Cai (University of British Columbia) started the second day by presenting his group's paper "A Wired Router Can Eliminate 802.11 Unfairness, But It's Hard." The key idea is that the wireless router and TCP, not the
802.11 medium-access control layer, would determine the bandwidth allocation. No change to 802.11 is thus required, so the scheme should be easy to deploy. The audience was interested in how much bandwidth might be wasted for the router-based allocation to achieve fairness, but the authors did not yet know the average throughput.

Because of the high costs associated with backhaul, network equipment, and spectrum, the deployment of wireless networks in urban environments is challenging. These costs are further aggravated by the infrastructure required for centralized user authentication. In this area, Sridhar Machiraju (Sprint) presented "Distributed Authentication for Low-Cost Wireless

\section{Drivers would reserve variable-sized slots on motorway lanes, which would enable enforcement of travel-time guarantees.}

Networks." He proposed a user authentication scheme that relies on base stations to collectively store authentication information, thus eliminating the need to maintain costly authentication servers and backhaul networks. The system distributes authentication by means of a unique token transferred between access points. The token contains the identity and information for whatever is being authenticated-for example, billing.

"Location-Based Trust for Mobile User-Generated Contents: Applications, Challenges, and Implementations" deals with how to trust the authenticity and quality of content that has been published by unknown individuals. Presented by Vincent Lenders (Princeton University), the paper proposes a secure localization and certification service that lets content producers tag their content with authenticated location and time information. One of the main features of this service is that it preserves producers' privacy by not exposing their identity to potential content consumers. However, it requires content producers' physical presence to tag created content and tie it to a location.

\section{OPERATING SYSTEMS AND DISTRIBUTED SYSTEMS}

Even after two decades of research in mobile computing, using mobile applications is frustrating because they can't adapt effectively to changes-for instance, in connectivity, remaining battery life, and user behavior. So said the authors of "quFiles: A Unifying Abstraction for Mobile Data Management," which was presented by Kaushik Veeraraghavan (University of Michigan). To solve these problems and simplify mobile data access and management, the authors propose a unifying file system abstraction called quFiles, which bundles arbitrary data types under a single name and gives requestors an appropriate data type and version. This results from an application-specific policy that considers information such as the platform, external devices, and context (for example, mobile device capabilities, network connectivity, and battery state). Veeraraghavan received the Best Student Presentation Award for his work.

"The Obscure Nature of Epidemic Quorum Systems," presented by João Barreto (INESC-ID and Technical University of Lisbon), argued that classical quorum systems aren't suitable for mobile and weakly connected environments. Other researchers recently proposed epidemic vote propagation to solve this problem, but the authors of this paper showed that epidemic quorum systems aren't always better than classical ones for such networks.

We can migrate a mobile user's computational environment between hosts by suspending the virtual machine (VM) on the source host, transferring its state, and resuming it on the 
destination. In "Low-Bandwidth VM Migration via Opportunistic Replay," Ajay Surie (Carnegie Mellon University) presented a method for optimizing the transfer of large amounts of disk and memory state during this process. Between frequently visited sites, the opportunistic-replay approach first captures a complete log of user interactions (such as keystrokes and mouse movements), then relays this $\log$ to the remote site. To alleviate the problem of potentially divergent VM states, the system uses cryptographic hashing techniques to identify and transmit only the differences. The authors received encouraging results from an early prototype that shows savings of up to 80.5 percent of bytes transferred.

\section{DEALING WITH SCREENS}

Chandrasekhar Narayanaswami (IBM T.J. Watson Research Center) presented a paper titled "Pervasive Symbiotic Advertising." He first outlined trends in online advertising and then extrapolated how this might apply to pervasive computing. In pervasive symbiotic advertising, targeted ads are delivered on public displays on the basis of a plethora of personal information from personal devices, but without intruding on the user's personal display. Narayanaswami then presented Celadon, a system that delivers business services such as advertising to mobile device users and large displays in public spaces. Celadon combines information about user preferences, product data, and availability of displays to provide interactive advertisements that connect to underlying services in a customized way.

John Collomosse (University of Bath) presented "Screen Codes: Visual Hyperlinks for Displays.” The display he described would show an image that slowly "sparkles." The main idea is to transmit information (such as hyperlinks) to camera phones simply by placing the camera phone in front of a display. A practical constraint for the work was that the CPUs in mobile phones don't have floating-point arithmetic in hardware, making them slow in signal processing. Audience members also suggested that perhaps this could be implemented with RFID.

A CAPTCHA is a type of challengeresponse test used to determine if a requestor is human. It can sometimes be hard for humans to solve. It also requires a keyboard, which can be hard to use on devices such as mobile phones. Philippe Golle (Palo Alto Research Center) presented a talk entitled "Making CAPTCHAs Clickable," which discussed how to convert regular keyboard-entry CAPTCHAs into clickable ones. A user study showed that solving clickable CAPTCHAs is only 52.2 percent slower on a cell phone screen than on a computer screen, while solving regular CAPTCHAs is 282 percent slower.

\section{DOCTORAL CONSORTIUM}

After the actual HotMobile program, a doctoral consortium was organized by Jason Hong (Carnegie Mellon University). This year, IBM sponsored registration for three students to the consortium and the HotMobile workshop. The students were guided by Deborah Estrin (University of California, Los Angeles), Christine Julien (University of Texas at Austin), Ranveer Chandra (Microsoft Research), and Victor Bahl (Microsoft Research).

Janne Lindqvist (Helsinki University of Technology, Finland) presented his research on privacy-enhancing technologies for wireless networks and the Internet. Lindquist's doctoral thesis work has included systematic analysis of information leaks from modern mobile computers, preventing these leaks, and privacy-preserving accesspoint discovery for 802.11.

Pravin Pawar (University of Twente, the Netherlands) presented his ongoing research on "Context-Aware Mobile Service Platform for Vertical Handover Support to Nomadic Mobile Services." His $\mathrm{PhD}$ work mainly involves apply- ing context-aware computing for the services hosted on mobile devices to improve their availability, responsiveness, and data resiliency.

Erich Stuntebeck (Georgia Institute of Technology) presented his research on the development of new systems and methods for indoor localization. His research focuses on the reuse of existing building infrastructures, such as in-wall electrical wiring, as part of the location system in order to minimize deployment burden and cost.

The doctoral consortium ended with discussion on possible improvements to the participating students' work, as well as suggestions about finishing $\mathrm{PhDs}$ in a timely manner and seeking career opportunities in academia and industrial research labs. P

Janne Lindqvist is a specialist researcher and $\mathrm{PhD}$ candidate in the Department of Computer Science and Engineering at Helsinki University of Technology, Finland. Contact him at janne.lindqvist@tml.hut.fi.

Pravin Pawar is a $\mathrm{PhD}$ candidate in the Remote Monitoring and Treatment Group, Department of Electrical Engineering, Mathematics and Computer Science, at the University of Twente, the Netherlands. Contact him at P.Pawar@ewi.utwente.nl.

Erich Stuntebeck is a PhD candidate in the College of Computing's Ubiquitous Computing Research Group at Georgia Institute of Technology. Contact him at
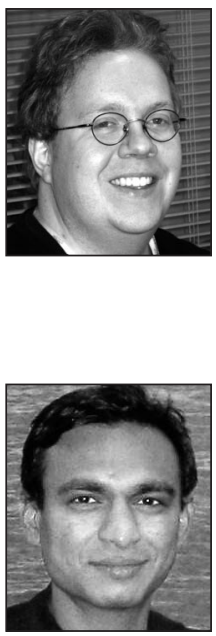
eps@gatech.edu. 